\title{
Interleukin-10 deficiency aggravates kidney inflammation and fibrosis in the unilateral ureteral obstruction mouse model
}

\author{
Yuanmeng Jin ${ }^{1,5}$, Ruijie Liu ${ }^{2,3,5}$, Jingyuan Xie ${ }^{1}$, Huabao Xiong ${ }^{4}$, John Cijiang He ${ }^{2,3}$ and Nan Chen ${ }^{1}$
}

Interleukin-10 functions as a general immunosuppressive cytokine, which also negatively regulates inflammatory responses through complex mechanisms. Recent studies suggested that IL-10 may also inhibit fibrosis in various diseased models. However, the role of IL-10 in renal fibrosis has not been demonstrated. Here, we investigated the effects of IL-10 in the development of renal tubulointerstitial fibrosis by creating the unilateral ureteral obstruction (UUO) model in IL-10 knockout ( / / ) mice. We performed sham or unilateral ureteral obstruction surgery in 8-week-old IL-10 - / male mice and age and sex-matched wild type littermates. Mice were killed at 7 days or 14 days post surgery and renal tissues were obtained for RNA, protein, and immunohistochemical analysis. Our results found IL-10 deficiency resulted in enhanced renal fibrosis demonstrated by more severe tubular injury and collagen deposition and higher expression of pro-fibrotic genes (including $\alpha$-SMA, MMP-2, fibronectin, FSP-1 and vimentin). Our results also found IL-10 - / - UUO mice developed more severe renal inflammation with a significant increase in inflammatory cells infiltration, and upregulation of inflammatory chemokines (MCP-1 and RANTES), and cytokines (TNF- $\alpha$, IL-6, IL-8, and M-CSF). Further study revealed that enhanced renal inflammation and fibrosis was associated with significantly increased activation of both TGF- $\beta /$ Smad 3 and NF- $\kappa$ B signaling pathways. In summary, our study provides the direct evidence that IL-10 is an endogenous cytokine that has a key role in protecting against development of renal inflammation and fibrosis. Enhancement of IL-10 expression could be a potential anti-fibrosis therapy for patients with chronic kidney diseases. Laboratory Investigation (2013) 93, 801-811; doi:10.1038/labinvest.2013.64; published online 29 April 2013

KEYWORDS: fibrosis; inflammation; interleukin-10; kidney; unilateral ureteral obstruction

Interleukin-10 (IL-10) is a key immunosuppressive cytokine produced by regulatory $\mathrm{T}$ cells and other cells. ${ }^{1}$ Its main biological function is to limit innate as well as adaptive immune responses and to protect host from immunemediated tissue damage. ${ }^{2,3}$ However, further studies reveal that IL-10 also has pivotal roles in suppressing inflammatory processes, mainly through blocking activation of inflammatory pathways and inhibiting the secretion of a broad spectrum of pro-inflammatory mediators, like IL-1, IL-6, IL-8, IL-12, interferon- $\gamma$ (IFN- $\gamma$ ), tumor necrosis factor- $\alpha$ (TNF- $\alpha)$, chemokine monocyte chemoattractant protein-1 (MCP-1), and macrophage inflammatory protein (MIP)-1, and IL-8. ${ }^{4-7}$
In addition, IL-10 has been recently shown to inhibit fibrosis in various animal models. IL-10 treatment could attenuate the severity of liver fibrosis induced by carbon tetrachloride (CCl4) in vivo. ${ }^{8}$ Several clinical studies also showed that IL-10 was able to reverse liver fibrosis without increasing viral titers in patients with chronic hepatitis C infection. ${ }^{9}$ The role of IL-10 in fibrosis has been also reported in other organs such as lung, heart and pancreas fibrosis. ${ }^{10-12}$ The underlying mechanism of antifibrotic effects of IL-10 remains unclear, but it has been suggested that IL-10 can suppress extracellular matrix synthesis and inhibit key inflammatory pathways such as NF- $\kappa$ B. ${ }^{13-15}$

\footnotetext{
${ }^{1}$ Department of Nephrology, Ruijin Hospital, Shanghai Jiao Tong University School of Medicine, Shanghai, PR China; ${ }^{2}$ Division of Nephrology, Department of Medicine, Mount Sinai School of Medicine, New York, NY, USA; ${ }^{3}$ James J. Peters Veteran Administration Medical Center, New York, NY, USA and ${ }^{4}$ Immunobiology Center, Mount Sinai School of Medicine, New York, NY, USA

Correspondence: Dr JC He, Division of Nephrology, Department of Medicine, Mount Sinai School of Medicine, One Gustave L Levy Place, Box 1243, New York, NY 10029, USA. Email: cijiang.he@mssm.edu or Dr N Chen, Department of Nephrology, Ruijin Hospital, Shanghai Jiao Tong University School of Medicine, No 197 Rui Jin Er Road, 200025 Shanghai, China. Email: chen-nan@medmail.com.cn
}

${ }^{5}$ These authors contributed equally to this work.

Received 18 September 2012; revised 13 March 2013; accepted 31 March 2013 
In the context of kidney, IL-10 has been reported to effectively suppress the progression of acute and chronic renal damage in vivo. ${ }^{16-22}$ However, the role of IL-10 in renal tubulointerstitial fibrosis has not been studied. Here, we used IL-10 - / - mice ${ }^{23-26}$ with unilateral ureteral obstruction (UUO), a well-established model for kidney fibrosis, to investigate the roles of IL-10 in the progression of renal fibrosis.

\section{MATERIALS AND METHODS}

\section{Animals and Experimental Protocol}

IL-10 - / - mice on C57BL/6 background obtained from Jackson Laboratory (Bar Harbor, Maine, CT, USA) were bred in the laboratory animal center at the Shanghai Jiao Tong University, School of Medicine. Male IL-10 - / - and wildtype (WT) littermates on B6 background ( $n=6$ /group) at 8 weeks of age underwent left ureteral obstruction or had sham operation. For mice in the UUO group, the left ureter was exposed through a mid-abdominal incision and ligated using 4-0 silk. All surgeries were performed under general anesthesia with isoflurane. All procedures were performed in accordance with the guidelines established by National Research Council Guide for the Care and Use of Laboratory Animals and approval of our Institute Animal Care and Use Committee (IACUC). Sham and UUO kidneys were harvested at day 7 or day 14 post-surgery.

\section{RNA Isolation and Quantitative Real-Time PCR}

Total RNAs was isolated from mouse kidney using the RNeasy mini kit (Qiagen, Hilden, Germany) according to the manufacturer. RT-PCR was carried out using the Bio-Rad iScript cDNA Synthesis kit. Quantitative real-time PCR with SYBR green PCR Master Mix (Applied Biosystems, Foster City, CA, USA) was performed in a MicroAmp Fast Optical 384-Well Reaction Plate (Applied Biosystems) using the 7300 Fast Real-Time PCR System (Applied Biosystems) for thermal cycling and real-time fluorescence measurements. Lightcycler analysis software was used for determining crossing points based on the second derivative method. Data were analyzed by the $2^{-\Delta \Delta \mathrm{CT}}$ methods and presented as fold change relative to a control sample after normalization against the expression of housekeeping genes. The sequences of the primer sets are given in Table 1.

\section{Western Blot Analysis}

Protein from homogenized frozen kidney was prepared in a lysis buffer. After sonication and centrifugation, supernatants were collected, and the protein concentration was determined with the BCA protein assay kit (Pierce, Rockford, IL, USA). Western blotting was performed as previously described. ${ }^{22,30}$ The primary antibodies were obtained from the following sources: anti-phospho-p65 (no. 3039), anti-phospho-Smad3 (no. 9520), anti-glyceraldehyde-3-phosphate dehydrogenase (no. 2118) (Cell Signaling Technology, Danvers, MA, USA). Anti-rabbit antibody conjugated with horseradish peroxidase
Table 1 Sequences of the real-time PCR Primers

\begin{tabular}{|c|c|c|}
\hline Genes & Sense primers $\left(5^{\prime}-3^{\prime}\right)$ & Anti-sense primers $\left(5^{\prime}-3^{\prime}\right)$ \\
\hline$\alpha-S M A$ & GCTGGTGATGATGCTCCCA & GCCCATTCCAACCATTACTCC \\
\hline Fibronectin & GATGCCGATCAGAAGTTGG & GGTTGTGCAGATCTCCTCGT \\
\hline MMP-2 & ACCCAGATGTGGCCAACTAC & GAGCAAAGGCATCATCCACT \\
\hline FSP-1 & CAGGCAAAGAGGGTGACAAG & TGCAGGACAGGAAGACACAG \\
\hline Vimentin & CGGCTGCGAGAGAAATTGC & CCACTITCCGTTCAAGGTCAAG \\
\hline Collagen I & AGCTITGTGGATACGCGGAC & TAGGCACGAAGTTACTGCAAG \\
\hline TNF- $\alpha$ & GACGTGGAACTGGCAGAAGAG & TTGGTGGTTGTGAGTGTGAG \\
\hline RANTES & GAGTGACAAACACGACTGCAAGAT & CTGCTTTGCCTACCTCTCCCT \\
\hline$\|-6$ & CCAGAAACCGCTATGAAGTTCCT & CACCAGCATCAGTCCCAAGA \\
\hline M-CSF & CCCATATTGCGACACCGAA & AAGCAGTAACTGAGCAACGGG \\
\hline$\|-8$ & CAGCTGCCTTAACCCCATCA & CTTGAGAAGTCCATGCGAAA \\
\hline MCP-1 & GACCCGTAAATCTGAAGCTAA & CACACTGGTCACTCCTACAGAA \\
\hline IL-10 & GCCTTCAGTATAAAAGGGGGACC & GTGGGTGCAGTTATTGTCTTCCCG \\
\hline GAPDH & CAGGGCTGCTTITAACTCTGGTAA & GGGTGGAATCATATTGGAACATGT \\
\hline
\end{tabular}

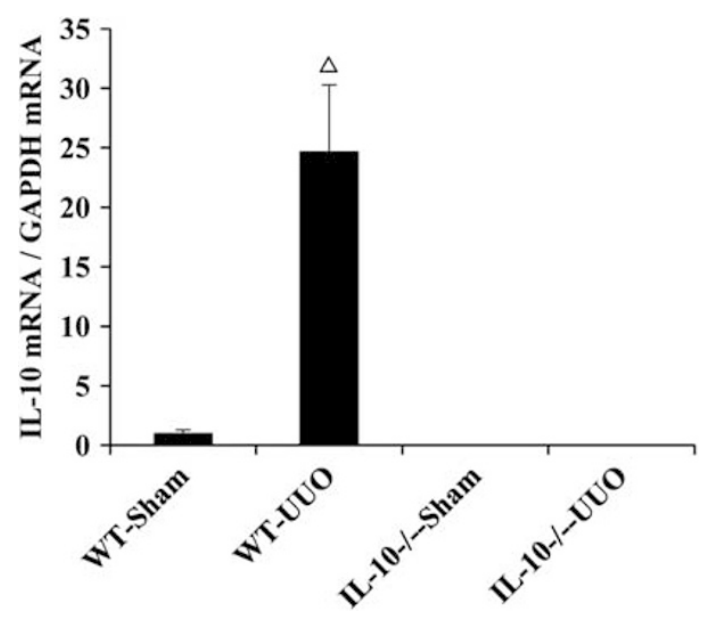

Figure 1 Determination of IL-10 mRNA levels in mice kidney by real-time PCR. IL-10 mRNA levels were increased markedly in WT mouse kidney after UUO. However, IL-10 mRNA levels were undetectable in IL-10deficient mice. Relative mRNA levels (fold induction over sham controls) were reported after normalization with GAPDH, ${ }^{\Delta} P<0.01, n=5$.

Table 2 Score of tubular injury

\begin{tabular}{lcccc}
\hline Mice & $\begin{array}{l}\text { WT UUO } \\
\text { (7 days) }\end{array}$ & $\begin{array}{c}\text { WT UUO } \\
(14 \text { days })\end{array}$ & $\begin{array}{c}\text { IL-10 }-/- \\
\text { UUO }(7 \text { days })\end{array}$ & $\begin{array}{c}\text { IL-10 }-/- \text { UUO } \\
(14 \text { days })\end{array}$ \\
\hline $\begin{array}{l}\text { Kidney injury } \\
\text { scoring }\end{array}$ & $1.8 \pm 0.8$ & $2.4 \pm 0.5$ & $3.2 \pm 0.7^{*}$ & $3.6 \pm 0.5^{*}$ \\
& & & &
\end{tabular}

IL-10-deficient mice developed more severe tubular injury after UUO than WT mice. The table summarizes the semiquantitative score of tubular injury in WT and IL-10 - / - mice after UUO. ${ }^{*} P<0.05$ as compared with WT UUO mice at 7 days or 14 days post-UUO, respectively, $n=6$. 
Picrosirius red staining
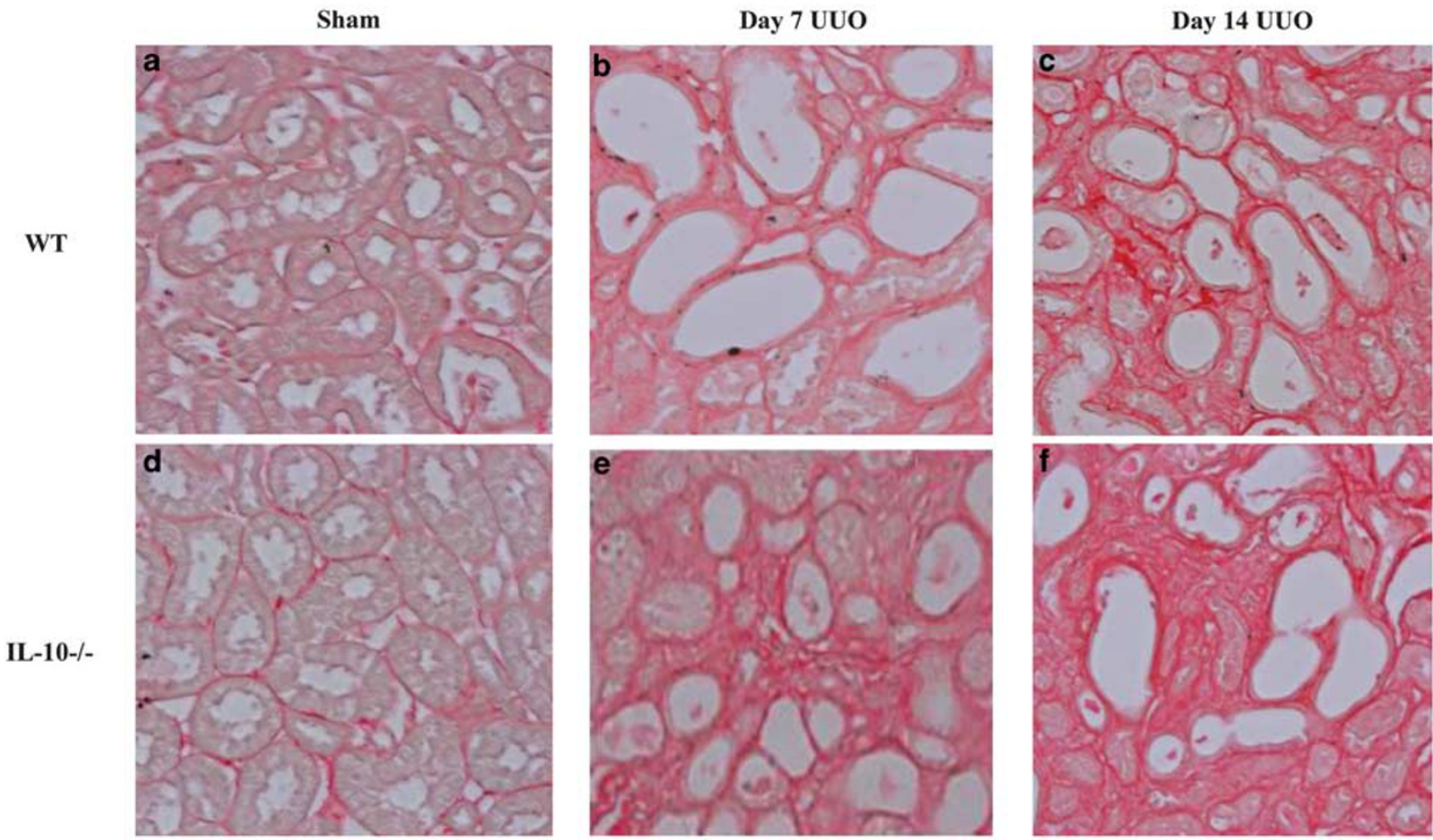

IL-10-/-

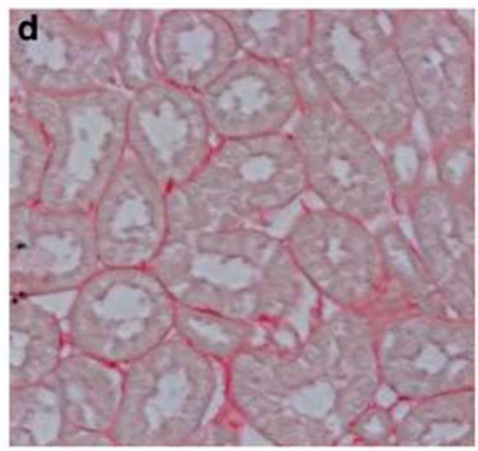

Sham
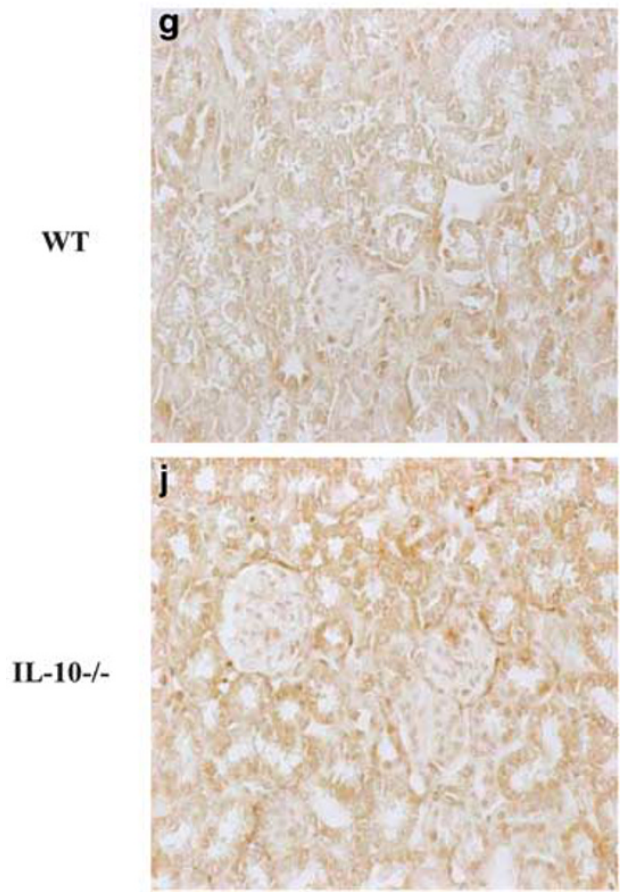

\section{Collagen}

Day 7 UUO

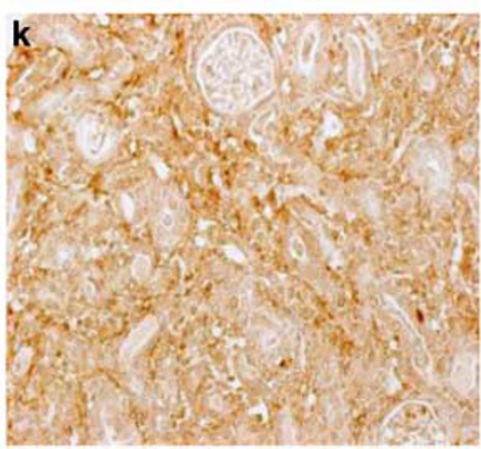

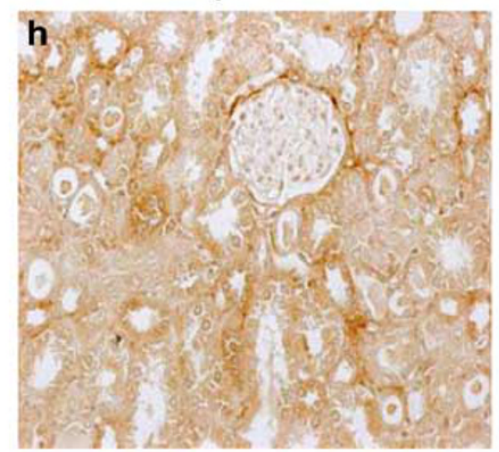

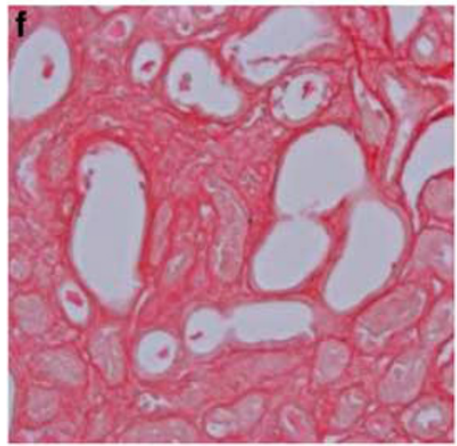

Day 14 UUO
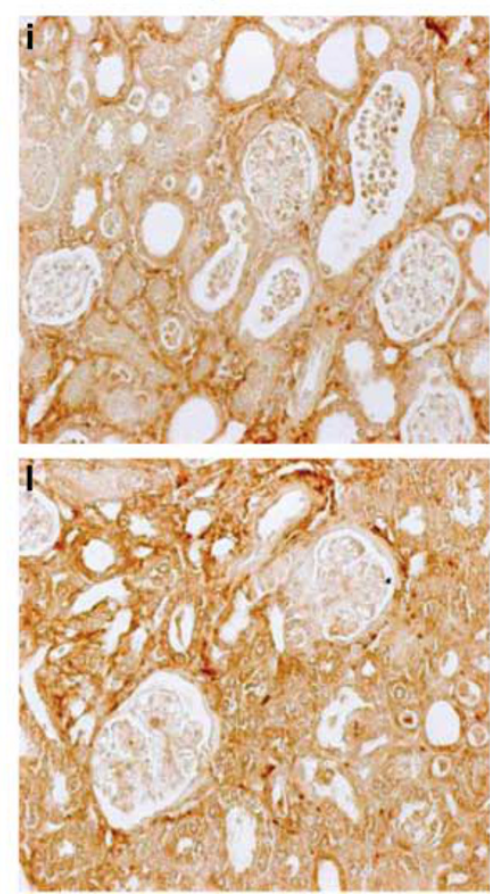

Figure 2 IL-10 deficiency increases renal tubulointerstitial collagen deposition after UUO. The graphs (a-f) and graphs (g-l) show representative images of collagen deposition stained with picrosirius red and anti-collagen I antibody in mice kidney (magnification $\times 200$ ). The graph (m) and $(\mathbf{n})$ summarize the results from quantification of these images by computer-assisted image analysis, expressed as percentage of the positive area. ${ }^{*} P<0.05$, ${ }^{\Delta} P<0.01$. 

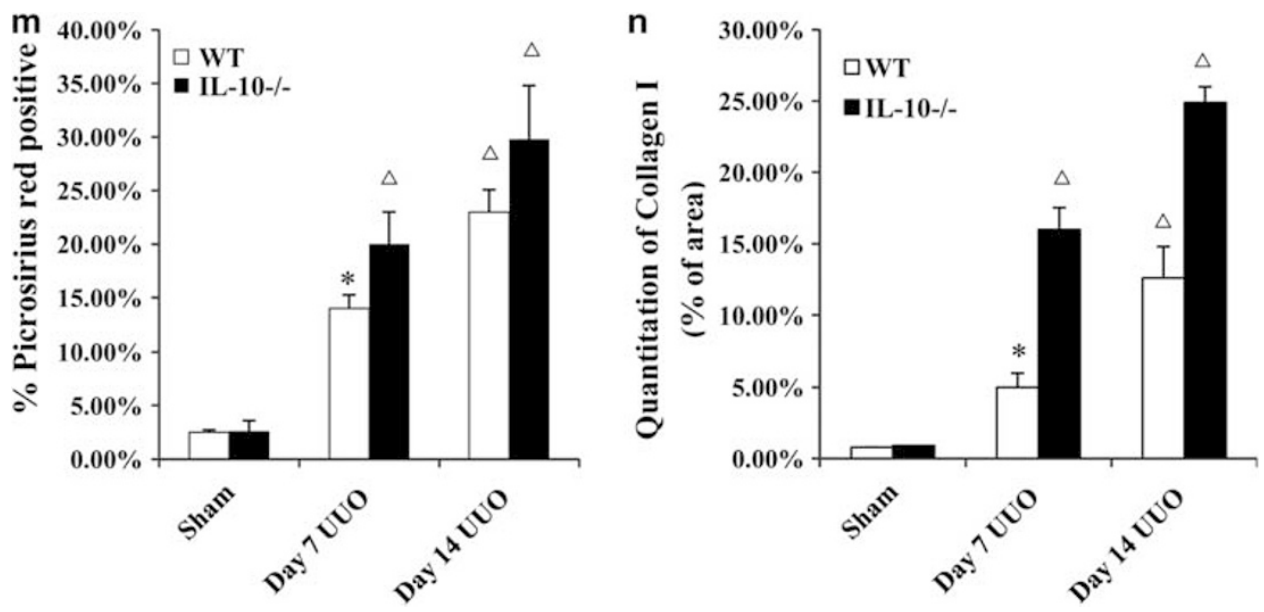

Figure 2 Continued.
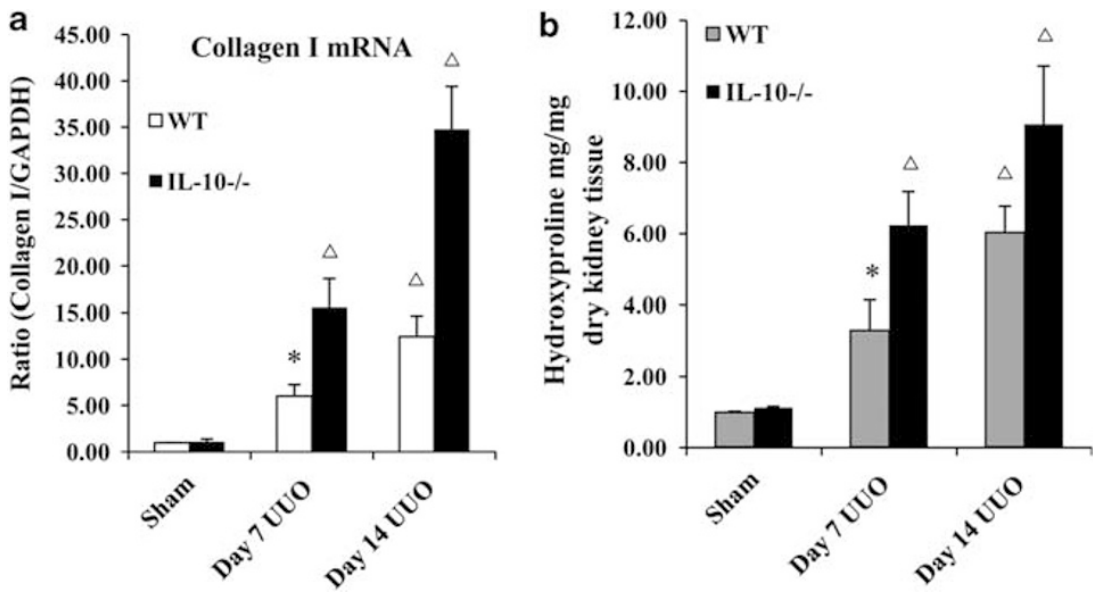

Figure 3 IL-10 deficiency accelerates collagen synthesis after UUO. The graph (a) summarizes analysis of relative collagen I mRNA expression (fold induction over sham controls) normalized to GAPDH, UUO relative to contralateral, by semiquantitative real-time PCR. The graph (b) shows total kidney collagen content measured by the hydroxyproline assay is significantly increased in obstructed kidneys from IL-10 - / - mice compared to WT UUO mice. ${ }^{*} P<0.05,{ }^{\Delta} P<0.01$.

was used as secondary antibody, and visualized using chemiluminescent substrate (SuperSignal; Pierce Chemical Co.). Quantification was performed by measuring the intensity of the bands with the use of the National Institutes of Health Image analysis software.

\section{Histology and Immunohistochemistry}

Histology scoring was performed by examining PAS-stained kidney sections of six mice in each group by an experienced pathologist on coded slides. The percentage of injured tubules in the cortex was estimated using a four-point scale by using the following criteria: tubular dilatation, epithelial simplification, and interstitial expansions in 10 randomly chosen, non-overlapping fields $(\times 400)$. Lesions were graded on a scale from 0 to 4 : $0=$ normal; $1=$ mild, involvement of $<25 \%$ of the cortex; $2=$ moderate, involvement of $25-50 \%$ of the cortex; $3=$ severe, involvement of $50-75 \%$ of the cortex; $4=$ involving $>75 \%$ of the cortex.

Picrosirius Red staining was performed for assessment of collagen deposition as described. ${ }^{27}$ The amount of cortical fibrillary collagen was determined by observing areas stained with picrosirius red with a Zeiss Axioplan 2IE microscope. Twenty non-overlapping fields at $\times 400$ magnification from each section were analyzed in a blinded manner and quantified using a digital image analysis program (MetaMorph7.6). Results are expressed as a percentage of the area with picrosirius red staining.

Indirect immunoperoxidase procedure was performed using a microwave-based antigen retrieval technique. ${ }^{28}$ Paraffin-embedded sections were stained with polyclonal goat anti-Collagen I antibody (Southern Biotech, Birmingham, AL, USA), monoclonal rat anti-F4/80 antibody (AbD Serotec, Oxford, UK), polyclonal rabbit anti- $\alpha$-SMA antibody and 
monoclonal rabbit anti-CD3 antibody (Abcam, Cambridge, MA), and developed with DAB substrate kit (Vector Laboratories, Burlingame, CA) to produce a brown color. For determination of immunohistochemistry staining, stained sections were imaged using the Image Analysis System (AxioVision 4, Carl Zeiss, Germany).

\section{Hydroxyproline Assay}

Collagen content was assessed by hydroxyproline assay according to the method of Samuel CS. ${ }^{29}$ One-fourth of the obstructed or contralateral kidney was completely hydrolyzed in $1 \mathrm{ml} 6 \mathrm{~N} \mathrm{HCl}$ by incubation at $110^{\circ} \mathrm{C}$ for $18 \mathrm{~h}$, before being neutralized with $10 \mathrm{~N} \mathrm{NaOH}$ and lyophilized. The obtained
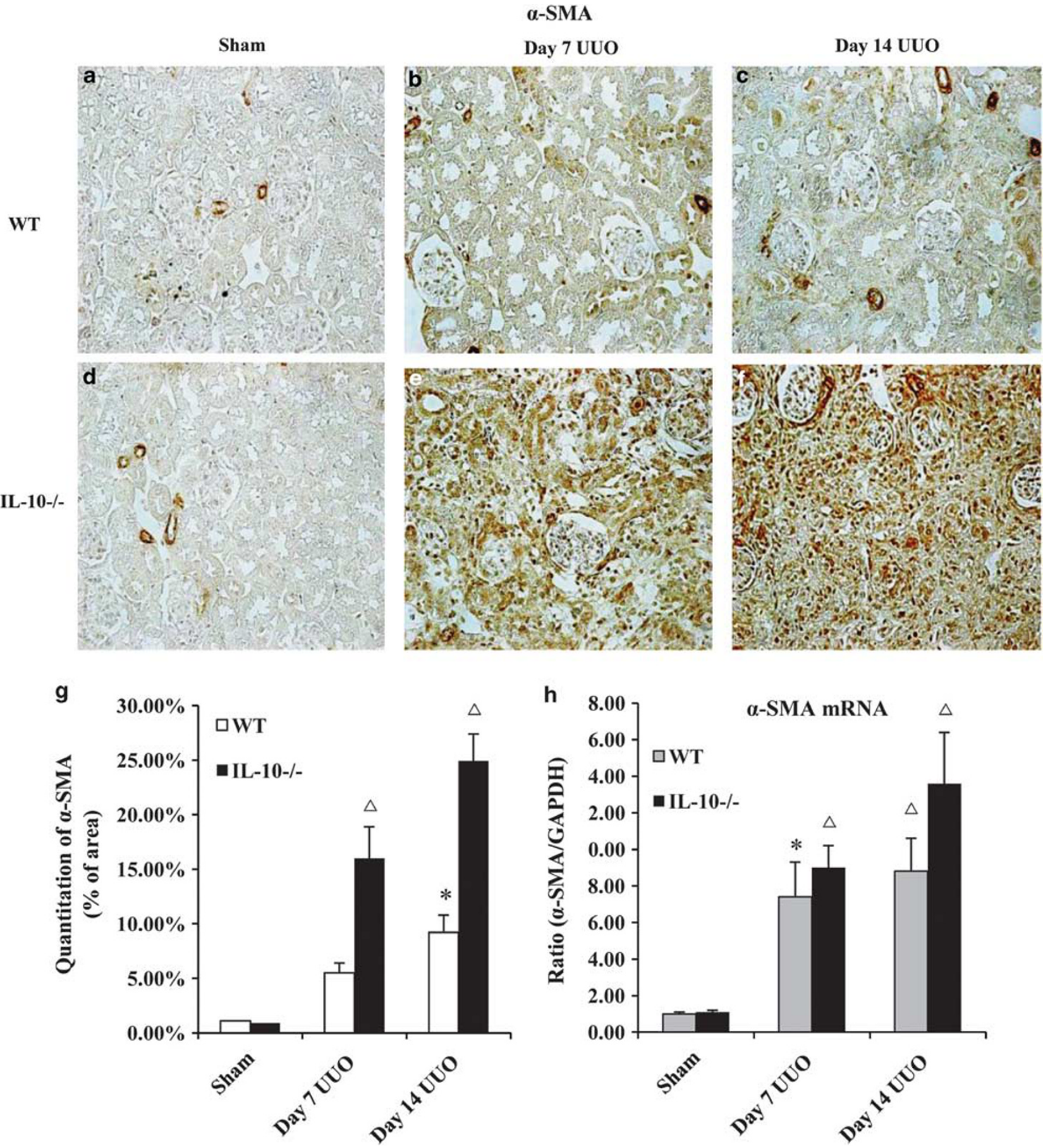

Figure 4 Deletion of IL-10 promotes $\alpha$-SMA expression in the obstructed kidney. Graph (a-f) shows representative images of $\alpha$-SMA + myofibroblast accumulation in mice kidney (magnification $\times 200$ ). Graph $(\mathbf{g})$ summarizes the results from quantification of these images by computer-assisted image analysis, expressed as percentage of the positive area. Graph (h) demonstrates that IL-10 deficiency significantly increased renal $\alpha$-SMA expression compared with WT UUO group. Relative $\alpha$-SMA mRNA (fold induction over sham controls) were reported after normalization with $\mathrm{GAPDH},{ }^{*} P<0.05,{ }^{\Delta} P<0.01$. 
dried residues were reconstituted with $0.1 \mathrm{M} \mathrm{HCl}$ and oxidized by chloramines- $\mathrm{T}$ for $4 \mathrm{~min}$ at room temperature. Then samples were mixed with p-dimethylaminonbenzaldehyde and placed for $25 \mathrm{~min}$ in a shaking water bath at $60^{\circ} \mathrm{C}$. The absorbance was measured at $558 \mathrm{~nm}$ using a spectrophotometer. Hydroxyproline content was calculated from the standard curve constructed with cis-4-hydroxy-L-proline (Sigma). The results were expressed as total collagen/kidney tissue, assuming that collagen contains an average of $14.4 \%$ hydroxyproline.

\section{Statistical Analysis}

All data are presented as mean \pm standard deviation $(X \pm$ s.d.). A nested ANOVA was used for all semiquantitative computer-assisted image analysis. For image analysis data, the arithmetic mean of six randomly selected images of slides for each animal was used to calculate the reported mean of the group and the s.d. All other results were analyzed by unpaired $t$-test between two groups after determination of data distribution. The Bonferroni correction was used when more than two groups were present. $P<0.05$ was considered statistically significant.

\section{RESULTS}

\section{IL-10 Expression in Mouse Kidney Tissues}

We firstly examined the mRNA levels of IL-10 in mouse kidneys of different groups by real-time PCR. As shown in Figure 1, IL-10 mRNA levels were nearly 25 -fold higher in kidneys of WT UUO mice than those of control mice. However, IL-10 mRNA levels were undetectable in IL-10deficient mouse kidneys. These data suggest that the endogenous anti-inflammatory cytokine IL-10 is upregulated in UUO kidneys. We also confirmed here the knockout of IL-10 gene in IL-10-deficient mice.

\section{IL-10 Deficiency Induces More Severe Tubular Injury in Obstructed Kidneys}

To study the role of IL-10 in the progression of renal fibrosis, we firstly analyzed tubular injury scores using PAS-stained kidney sections from these mice. As shown in Table 2, we found that WT mice demonstrated significant tubular injury after UUO. However, IL-10-deficient mice developed more severe tubular injury in the obstructed kidneys than WT mice at both 7 and 14 days after UUO.

\section{IL-10 Deficiency Promoted Renal Fibrosis in UUO Mice}

We first investigated the effects of IL-10 on the kidney collagen deposition. By specific staining of collagen I and real-time PCR, we found that both collagen protein accumulation (Figure 2) and mRNA expression (Figure 3a) were significantly increased in the obstructed kidneys of IL-10 - / mice compared with WT mice at each time point. This was further confirmed by quantification of the collagen content in the kidney as determined by measurement of hydroxyproline, a major component of collagen (Figure $3 \mathrm{~b}$ ).
We also determined the activation of myofibroblasts in the kidneys of these mice. We found more prominent increase in staining for $\alpha$-smooth muscle actin ( $\alpha$-SMA) (Figures $4 \mathrm{a}-\mathrm{g}$ ) and higher levels of $\alpha$-SMA mRNA (Figure $4 \mathrm{~h}$ ) in obstructed kidneys of IL-10 - / - mice as compared with those of WT mice. These results suggested that IL-10 deficiency induced more severe renal fibrosis.

\section{IL-10 Deficiency Induced Pro-Fibrotic Gene Expression in the UUO Kidney}

Next, we examined the expression of several pro-fibrotic markers in kidneys of these mice by real-time PCR. As shown in Figure 5, UUO caused a dramatic induction of the mRNA expression of pro-fibrotic genes, including fibronectin, matrix metalloproteinase-2 (MMP-2), fibroblast-specific protein-1(FSP-1) and vimentin, in the UUO kidneys than the sham-operated ones. The increase of these genes was much higher in IL-10 - / - UUO mice than WT UUO mice. These results suggest that IL-10 deficiency promotes key profibrotic genes expression in the UUO kidney.

\section{IL-10 Deficiency Induced Inflammatory Cells Infiltration and Inflammatory Cytokines Expression in the UUO Mice} Inflammation has a critical role in the renal tubulointerstitial fibrosis. To explore the potential mechanism involved in antifibrotic effects of IL-10 in the UUO model, we examined inflammatory response in both UUO and control mice. As shown in Figure 6, there were only a few of F4/80 + macrophages and $\mathrm{CD} 3+\mathrm{T}$ cells in kidneys of control groups. At day 7 and day 14 after UUO, infiltration of F4/80 + macrophages and $\mathrm{CD} 3+\mathrm{T}$ cells progressively increased in the interstitial areas of the obstructed kidneys more in IL-10 - / mice than in WT mice. We also examined the effects of

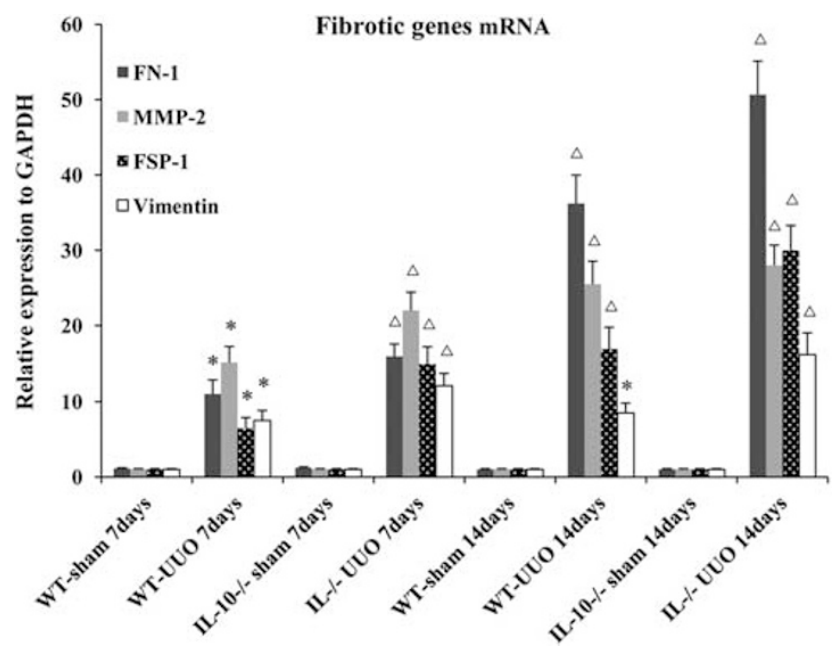

Figure 5 Deletion of IL-10 up-regulates fibrotic genes expression in the obstructed kidney. Representative real-time PCR analysis and quantitative data show that IL-10 deficiency increased renal MMP-2, fibronectin, FSP-1, and vimentin mRNA expression after obstructive injury. Relative mRNA levels (fold induction over sham controls) were reported after normalization with GAPDH, respectively. ${ }^{*} P<0.05, \Delta P<0.01$. 


\section{F4/80}
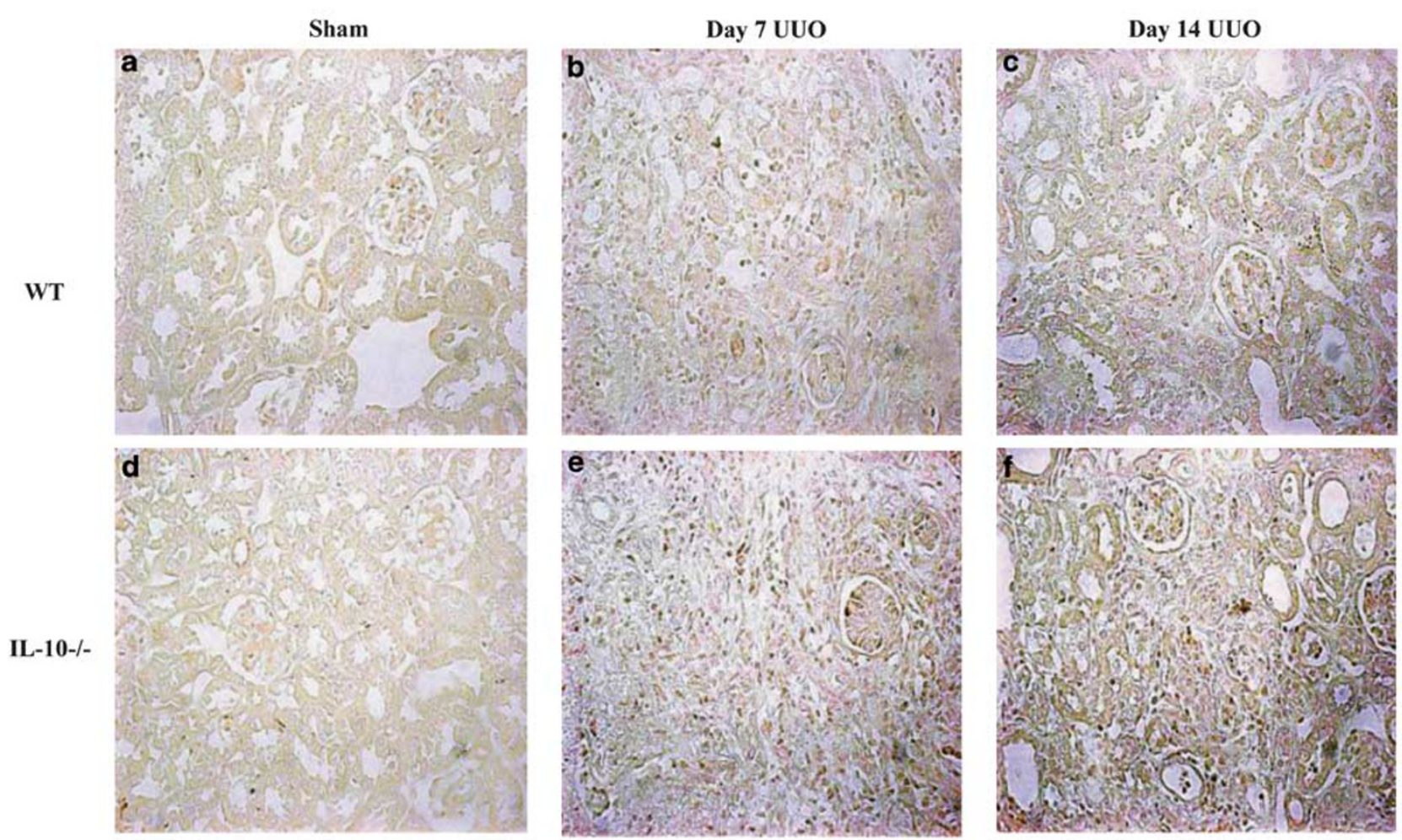

\section{CD3}
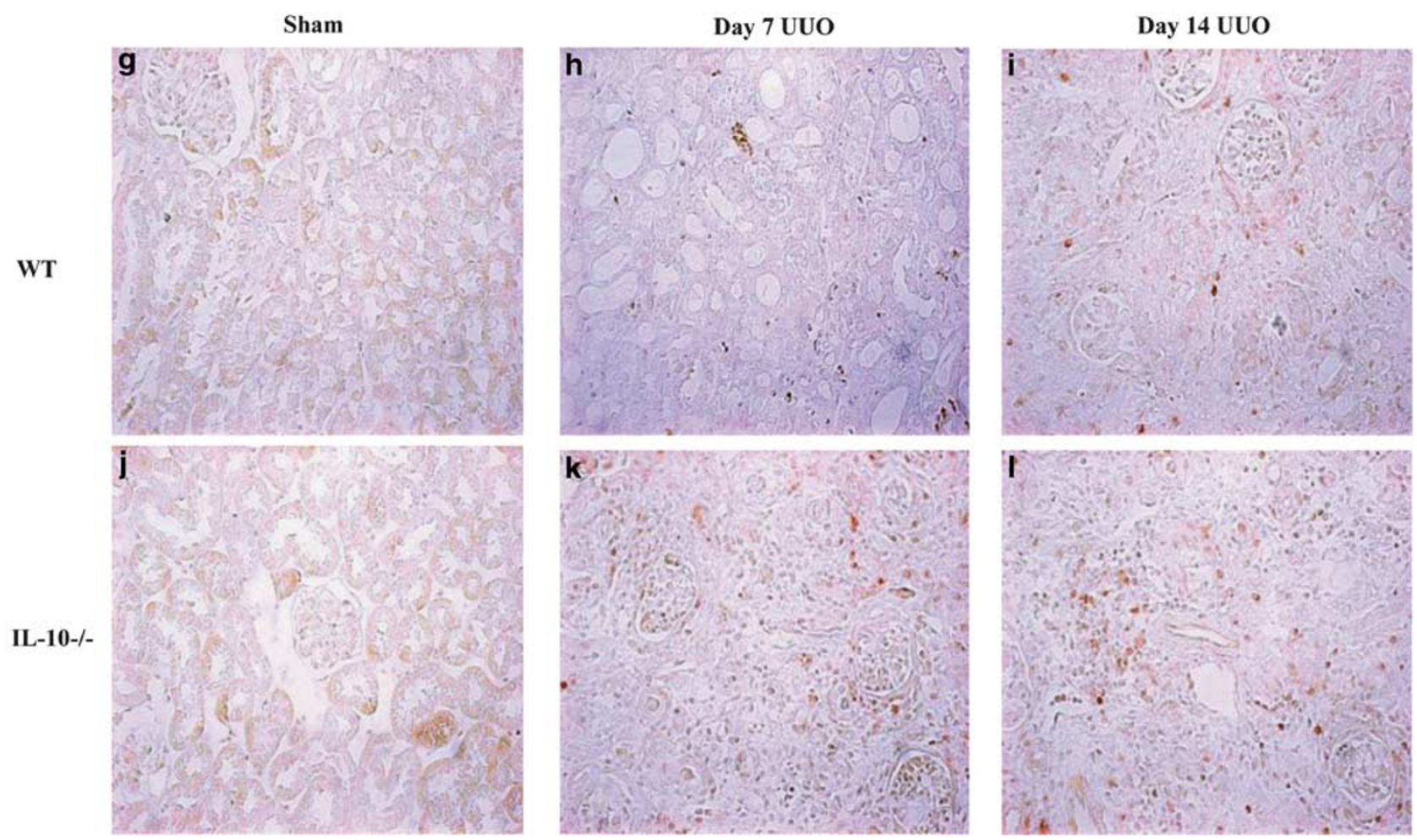

Figure 6 Deletion of IL-10 induces inflammatory cells infiltration in the obstructed kidney. Immunohistochemistry reveals that IL-10 deficiency enhances F4/80 + macrophage and CD3 + T cell infiltration in the UUO kidney. (a-f) F4/80. (g-I) CD3. The graph (m) and (n) show quantitative analysis of immunohistochemical staining. ${ }^{*} P<0.05,{ }^{\Delta} P<0.01$. 

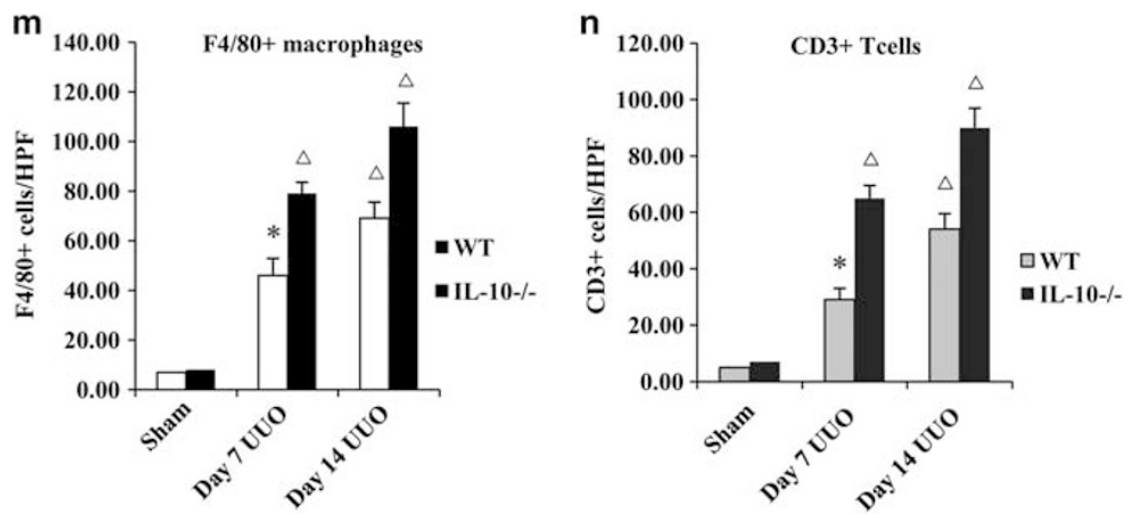

Figure 6 Continued.

IL-10 on the expression of chemokines, including MCP-1 and RANTES, as well as pro-inflammatory cytokines, including TNF-a, IL-6, IL-8 and macrophage-colony stimulating factor (M-CSF), which were important molecules that mediated macrophage and $\mathrm{T}$ cell chemotaxis. As shown in Figure 7, compared with WT groups, expression of MCP-1, TNF-a, IL-6, IL-8 and M-CSF were upregulated more in IL-10 - / mice at day 7 and day 14 after UUO. Expression of RANTES was also increased but there was no significant difference between WT UUO and IL-10 - / - UUO mice.

\section{IL-10 Deficiency Induces More Activation of Inflammatory and Fibrosis Pathways in the UUO Kidney}

To better understand potential mechanisms by which IL-10 regulated renal inflammatory and fibrotic response, we examined activities of TGF- $\beta$ and NF- $\kappa$ B pathways, which are known to have critical roles in the progression of renal fibrosis (Figure 8). We found phosphorylated Smad3 and p65 were weakly expressed in the kidneys of sham-operated mice. A marked increase of these phosphorylated proteins was observed in WT mice as early as 7 days after UUO. However, there was more increase of Smad3 and p65 phosphorylation in IL-10 - / - UUO mice than WT UUO mice at both 7 days and 14 days after UUO. These data suggests that IL-10 deficiency may significantly enhance the activation of key pro-inflammatory and pro-fibrosis pathways in the UUO kidney.

\section{DISCUSSION}

Renal tubulointerstitial fibrosis is the final common pathway leading to end-stage renal disease (ESRD) in various types of renal diseases and its severity correlates strongly with the inflammatory status. Elucidation of the primary mechanisms involved in tubulointerstitial fibrosis is an important step toward developing new therapies to prevent progressive kidney diseases resulting in ESRD. IL-10 is a general immunosuppressive cytokine and has been proved to negatively regulate inflammatory responses and inhibit fibrosis in var-

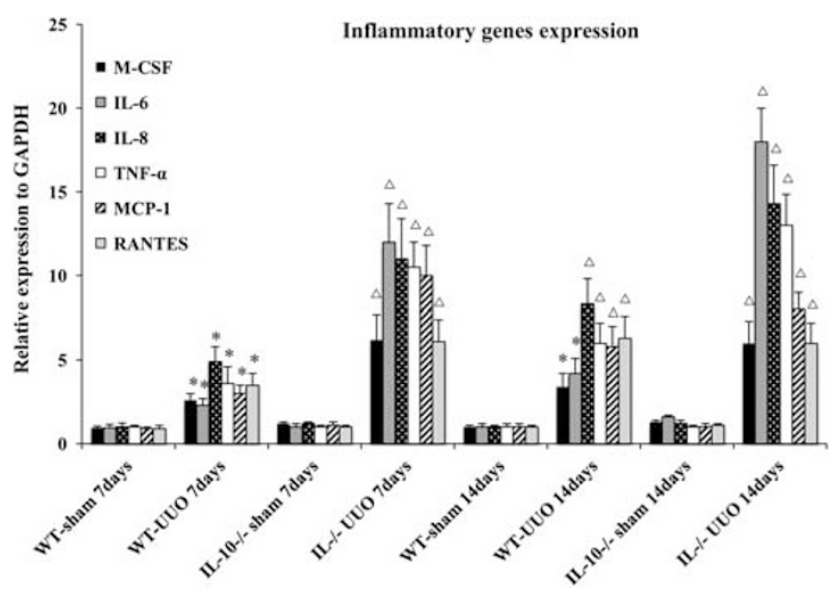

Figure 7 Deletion of IL-10 up-regulates inflammatory genes expression in the obstructed kidney. Representative real-time PCR analysis and quantitative data show that IL-10 deficiency increased mRNA expression of MCP-1, RANTES, TNF-a, IL-6, IL-8 and M-CSF after obstructive injury. Relative mRNA levels (fold induction over sham controls). were reported after normalization with GAPDH, respectively. ${ }^{*} P<0.05,{ }^{\Delta} P<0.01$.

ious diseased models. However, the relationship between IL10 and tubulointerstitial fibrosis is not fully understood.

The present study was the first one to examine the role of IL-10 in the process of interstitial fibrosis using the UUO model, which has been widely used as an animal model of tubulointerstitial disease. We found that IL-10 deficiency deteriorated tubular injuries and promoted renal fibrosis in the kidney of UUO mice. We also found IL-10 deficiency lead to more severe inflammatory cell infiltration (macrophage and $\mathrm{T}$ cells) and higher expression of pro-inflammatory cytokines (TNF- $\alpha$, IL-6, M-CSF, and IL-8) and chemokines (MCP-1, RANTES). Additionally, TGF- $\beta /$ Smad 3 and NF- $\kappa$ B pathways were activated more in IL-10 - / - UUO mice than in WT UUO mice. These findings provide evidence that IL-10 has a critical role in protecting against development of renal inflammation and fibrosis. 
a

$\frac{\text { Sham }}{\text { wide type }}+$ IL-10-/-
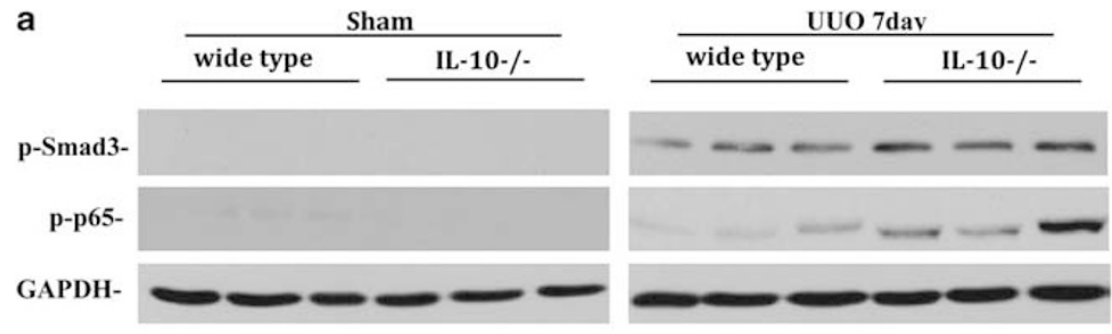

b
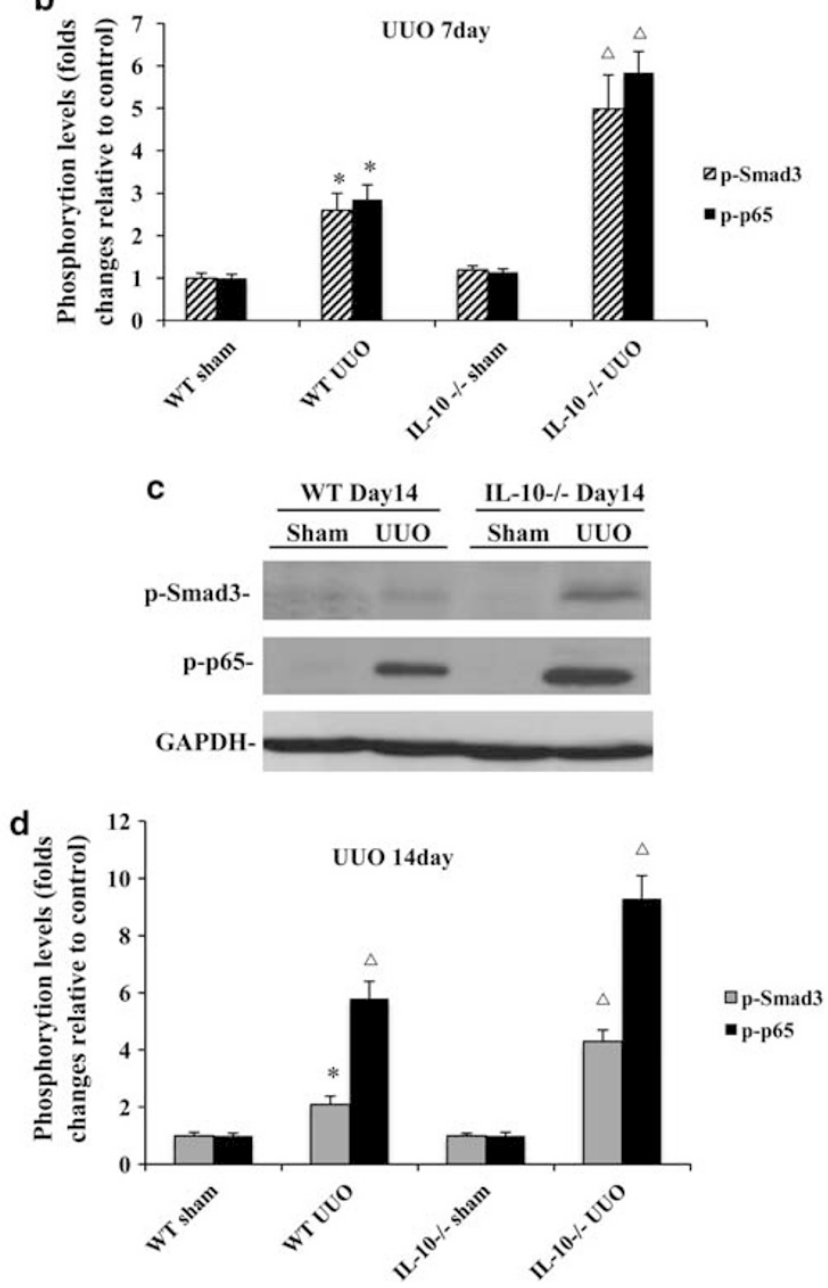

p-Smad3-

p-p65-

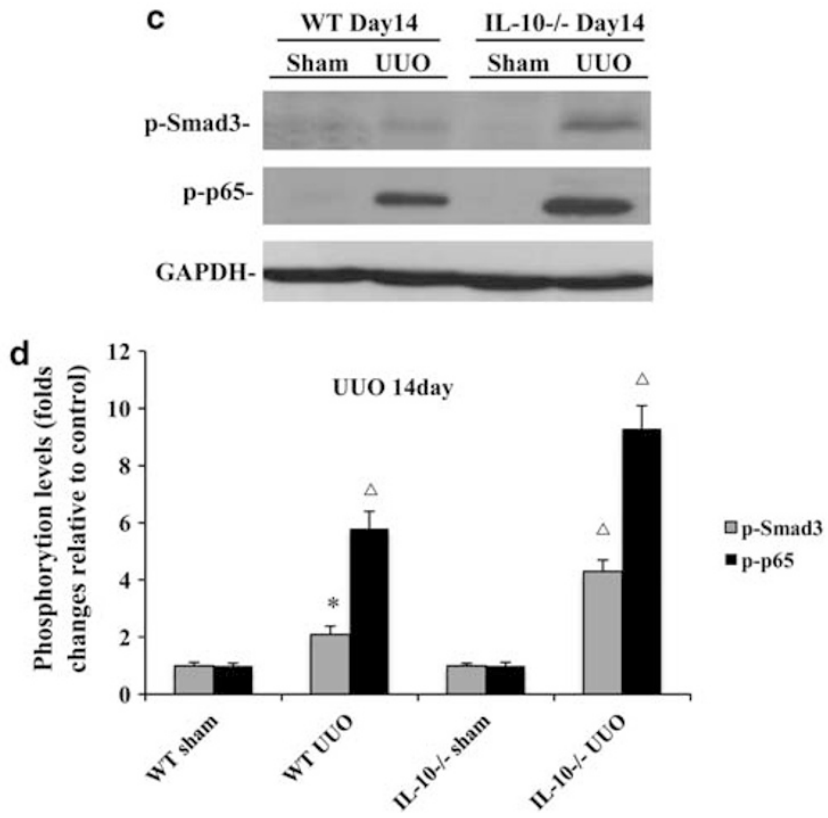

Figure 8 Deletion of IL-10 induces higher phosphorylation levels of Smad3 and p65 in the obstructed kidney. Representative Western blot analysis and quantitative data show much higher phosphorylation levels of these proteins in IL-10 - / - mice than WT mice at day 7(a and b) and day 14(c and d) after UUO. Relative protein levels (fold induction over sham controls) were reported after normalization with GAPDH, respectively. ${ }^{*} P<0.05,{ }^{\Delta} P<0.01$.

Our data are consistent with previous studies that demonstrated the reno-protective effects of IL-10 in vivo. Kitching et $a l^{16}$ and Choi et $a l^{17}$ found IL-10 therapy effectively reduced glomerular macrophage influx and expression of inflammatory molecules (IL- $1 \beta$, ICAM-1) in anti-Thyl-induced acute glomerulonephritis in rats and prevented the development of glomerulosclerosis in FGS/ Kist mice. Other studies confirmed that IL-10 administration could significantly reduce renal inflammatory cells infiltration in the interstitial areas of cortex, suppress chemokines (MCP-1, RANTES) and cytokines (IFN- $\gamma$, IL-2, iNOS, TNF- $\alpha$ ) expression, resulting in a less fibrotic response in models of chronic renal diseases induced by 5/6 nephrectomy ${ }^{19}$ and adriamycin treatment. ${ }^{20}$ Additionally, Semedo et $a l^{21}$ and Donizetti et a ${ }^{22}$ found IL-10 expression was negatively correlated with fibrotic genes expression (including vimentin, Collagen I, FSP-1) in remnant kidney model and unilateral ischemia model, indirectly suggesting a 
role of IL-10 in attenuating renal fibrosis. Studies in other organs, including lung, heart, pancreas and liver, also showed IL-10 could suppress inflammatory response and thereby inhibit matrix remodeling and fibrosis process, even if fibrosis had developed. ${ }^{31-37}$ Here, we provide direct evidence supporting the role of IL-10 in renal fibrosis by demonstrating that knockout of IL-10 leads to more severe inflammatory response and renal injury and fibrosis in the UUO model.

In addition, we found that knockout of IL-10 enhanced activation of TGF- $\beta / \mathrm{Smad} 3$ and NF- $\kappa \mathrm{B}$ pathways at days 7 and 14 after UUO, which are known to have central roles in the initiation and progression of renal inflammation and fibrosis through inducing a wide array of genes encoding pro-inflammatory cytokines, adhesion molecules, and chemokines in human and experimental models of kidney diseases. These results are also consistent with previous studies suggesting IL-10 mediates anti-inflammation and anti-fibrotic effects through negatively regulating these signaling pathways. ${ }^{38-44}$ It has been shown that the treatment with IL-10 could inhibit TGF- $\beta$ and NF- $\kappa$ B activation to reduce inflammatory response, ECM production and fibrotic genes expression in animal models of pulmonary and hepatic fibrosis. ${ }^{34,45}$

In summary, the present study suggests that the deficiency of IL-10 aggravates inflammation and fibrosis in obstructive kidney disease. This was associated with the loss of inhibitory effects of IL-10 on inflammatory pathways, including TGF- $\beta /$ Smad3 signaling pathway and NF- $\kappa$ B signaling pathway. Thus, IL-10 may be a critical negative regulator in renal inflammation and fibrosis. Results from this study further support the notion that IL-10 could be a therapeutic agent for kidney fibrotic diseases.

\section{ACKNOWLEDGEMENTS}

This work is the result of a collaborative effort between the Nephrology Department of Ruijin Hospital, Shanghai Jiao Tong University School of Medicine, China and the Nephrology Division of Mount Sinai Hospital, New York, USA. This work is supported by National Basic Research Program of China 973, program No.2012CB517600 (No.2012CB517604), National key technology R\&D Program (12-5), program NO. 2011BAl10B00 (2011BAl10B06), National Natural Science Foundation of China (No. 81070568), National Natural Science Foundation of China (No. 81000295) and National Natural Science Foundation of China (No. 81200526).

\section{DISCLOSURE/CONFLICT OF INTEREST}

The authors declare no conflict of interest.

1. Goldman $M$, Velu T. Interleukin-10 and its implications for immunopathology. Adv Nephrol Necker Hosp 1995;24:79-90.

2. Moore KW, de Waal Malefyt R, Coffman RL, et al. Interleukin-10 and the interleukin-10 receptor. Annu Rev Immunol 2001;19:683-765.

3. Asadullah K, Sterry W, Volk HD.. Interleukin-10 therapy-review of a new approach. Pharmacol Rev 2003;55:241-269.

4. De Waal Malefyt R, Haanen J, Spits H, et al. Interleukin 10 (IL-10) and viral IL-10 strongly reduce antigen-specific human T cell proliferation by diminishing the antigen-presenting capacity of monocytes via downregulation of class II major histocompatibility complex expression. J Exp Med 1991;174:915-924.
5. Fiorentino DF, Zlotnik $A$, Vieira $P$, et al. IL-10 acts on the antigenpresenting cell to inhibit cytokine production by Th1 cells. J Immunol 1991;146:3444-3451.

6. Ding L, Shevach EM.. IL-10 inhibits mitogen-induced $T$ cell proliferation by selectively inhibiting macrophage costimulatory function. J Immunol 1992;148:3133-3139.

7. Hsu DH, Moore KW, Spits H.. Differential effects of IL-4 and IL-10 on IL2-induced IFN-gamma synthesis and lymphokine-activated killer activity. Int Immunol 1992;4(5):563-569.

8. Chou WY, Lu CN, Lee TH, et al. Electroporative interleukin-10 gene transfer ameliorates carbon tetrachloride-induced murine liver fibrosis by MMP and TIMP modulation. Acta Pharmacol Sin 2006;27:469-476.

9. Nelson DR, Tu Z, Soldevila-Pico C, et al. Long-term interleukin 10 therapy in chronic hepatitis $C$ patients has a proviral and antiinflammatory effect. Hepatology 2003;38:859-868.

10. Arai T, Abe $\mathrm{K}$, Matsuoka $\mathrm{H}$, et al. Introduction of the interleukin-10 gene into mice inhibited bleomycin-induced lung injury in vivo. Am J Physiol Lung Cell Mol Physiol 2000;278:L914-L922.

11. Demols A, Van Laethem JL, Quertinmont E, et al. Endogenous interleukin-10 modulates fibrosis and regeneration in experimental chronic pancreatitis. Am J Physiol Gastrointest Liver Physiol 2002;282:G1105-G1112.

12. Nonaka-Sarukawa $M$, Okada $T$, Ito $T$, et al. Adeno-associated virus vector-mediated systemic interleukin-10 expression ameliorates hypertensive organ damage in Dahl salt-sensitive rats. J Gene Med 2008;10:368-374.

13. Fueki $\mathrm{N}$, Sagara $\mathrm{H}$, Akimoto $\mathrm{K}$, et al. Interleukin-10 regulates transforming growth factor-beta signaling in cultured human bronchial epithelial cells. Respiration 2007;74:454-459.

14. Schottelius AJ, Mayo MW, Sartor RB, et al. Interleukin-10 signaling blocks inhibitor of kappaB kinase activity and nuclear factor kappaB DNA binding. J Biol Chem 1999;274:31868-31874.

15. Asadullah K, Sterry W, Volk HD.. Interleukin-10 therapy-review of a new approach. Pharmacol Rev 2003;55:241-269.

16. Kitching $A R$, Katerelos $M$, Mudge $S J$, et al. Interleukin-10 inhibits experimental mesangial proliferative glomerulonephritis. Clin Exp Immunol 2002;128:36-43.

17. Choi YK, Kim YJ, Park HS, et al. Suppression of glomerulosclerosis by adenovirus-mediated IL-10 expression in the kidney. Gene Ther 2003;10:559-568.

18. Deng J, Kohda $\mathrm{Y}, \mathrm{Chiao} \mathrm{H}$, et al. Interleukin-10 inhibits ischemic and cisplatin-induced acute renal injury. Kidney Int 2001;60:2118-2128.

19. Mu W, Ouyang X, Agarwal A, et al. IL-10 suppresses chemokines, inflammation, and fibrosis in a model of chronic renal disease. J Am Soc Nephrol 2005;16:3651-3660.

20. Cao Q, Wang Y, Zheng D, et al. IL-10/TGF-beta-modified macrophages induce regulatory $\mathrm{T}$ cells and protect against adriamycin nephrosis. $J$ Am Soc Nephrol 2010;21:933-942.

21. Semedo P, Correa-Costa M, Antonio Cenedeze M, et al. Mesenchymal stem cells attenuate renal fibrosis through immune modulation and remodeling properties in a rat remnant kidney model. Stem Cells 2009:27:3063-3073.

22. Donizetti-Oliveira C, Semedo P, Burgos-Silva M, et al. Adipose tissuederived stem cell treatment prevents renal disease progression. Cell Transplant 2012;21:1727-1741.

23. Kühn R, Löhler J, Rennick $D$, et al. Interleukin-10-deficient mice develop chronic enterocolitis. Cell 1993;75:263-274.

24. Berg DJ, Kühn R, Rajewsky K, et al. Interleukin-10 is a central regulator of the response to LPS in murine models of endotoxic shock and the Shwartzman reaction but not endotoxin tolerance. J Clin Invest 1995;96:2339-2347.

25. Opal SM, Wherry JC, Grint P.. Interleukin-10: potential benefits and possible risks in clinical infectious diseases. Clin Infect Dis 1998;27:1497-1507.

26. Chevalier RL, Forbes MS, Thornhill BA.. Ureteral obstruction as a model of renal interstitial fibrosis and obstructive nephropathy. Kidney Int 2009;75:1145-1152.

27. Mor-Vaknin N, Punturieri A, Sitwala K, et al. Vimentin is secreted by activated macrophages. Nat Cell Biol 2003;5:59-63.

28. Lan HY, Mu W, Nikolic-Paterson DJ, et al. A novel, simple, reliable, and sensitive method for multiple immunoenzyme staining: use of microwave oven heating to block antibody crossreactivity and retrieve antigens. J Histochem Cytochem 1995;43:97-102. 
29. Samuel CS. Determination of collagen content, concentration, and sub-types in kidney tissue. Methods Mol Biol 2009;466: 223-235.

30. Mor-Vaknin N, Punturieri A, Sitwala K, et al. Vimentin is secreted by activated macrophages. Nat Cell Biol 2003;5:59-63.

31. Huang $\mathrm{YH}$, Shi MN, Zheng WD, et al. Therapeutic effect of interleukin10 on CCl4-induced hepatic fibrosis in rats. World J Gastroenterol 2006;12:1386-1391.

32. Demols A, Van Laethem JL, Quertinmont E, et al. Endogenous interleukin-10 modulates fibrosis and regeneration in experimenta chronic pancreatitis. Am J Physiol Gastrointest Liver Physiol 2002, 282:G1105-G1112.

33. Van Laethem JL, Eskinazi R, Louis $\mathrm{H}$, et al. Multisystemic production of interleukin 10 limits the severity of acute pancreatitis in mice. Gut 1998;43:408-413.

34. Garantziotis S, Brass DM, Savov J, et al. Leukocyte-derived IL-10 reduces subepithelial fibrosis associated with chronically inhaled endotoxin. Am J Respir Cell Mol Biol 2006;35:662-667.

35. Nakagome K, Dohi M, Okunishi K, et al. In vivo IL-10 gene delivery attenuates bleomycin induced pulmonary fibrosis by inhibiting the production and activation of TGF-beta in the lung. Thorax 2006; 61:886-894.

36. Krishnamurthy $\mathrm{P}$, Rajasingh J, Lambers $\mathrm{E}$, et al. IL-10 inhibits inflammation and attenuates left ventricular remodeling after myocardial infarction via activation of STAT3 and suppression of HuR. Circ Res 2009;104:e9-18.

37. Apparailly F, Verwaerde C, Jacquet C, et al. Adenovirus-mediated transfer of viral IL-10 gene inhibits murine collagen-induced arthritis. J Immunol 1998;160:5213-5220.

38. Anders HJ, Muruve DA.. The inflammasomes in kidney disease. J Am Soc Nephrol 2011;22:1007-1018.

39. Sanz AB, Sanchez-Niño MD, Ramos AM, et al. NF-kappaB in renal inflammation. J Am Soc Nephrol 2010;21:1254-1262.

40. Sanchez-Munoz F, Dominguez-Lopez A, Yamamoto-Furusho JK.. Role of cytokines in inflammatory bowel disease. World J Gastroenterol 2008; 14:4280-4288.

41. Kim EY, Moudgil KD.. Regulation of autoimmune inflammation by proinflammatory cytokines. Immunol Lett 2008;120:1-5.

42. Sanjabi S, Zenewicz LA, Kamanaka M, et al. Anti-inflammatory and proinflammatory roles of TGF-beta, IL-10, and IL-22 in immunity and autoimmunity. Curr Opin Pharmacol 2009;9:447-453.

43. Gordon JW, Shaw JA, Kirshenbaum LA.. Multiple facets of NF- $\kappa$ B in the heart: to be or not to NF- $\kappa$ B. Circ Res 2011;108:1122-1132.

44. Baker RG, Hayden MS, Ghosh S.. NF- $\kappa$ B, inflammation, and metabolic disease. Cell Metab. 2011;13:11-22.

45. Thompson K, Maltby J, Fallowfield J, et al. Interleukin-10 expression and function in experimental murine liver inflammation and fibrosis. Hepatology 1998;28:1597-1606. 